\title{
Level of articulation and short-term recognition following brief probe delays
}

\author{
DAVID J. MURRAY \\ Queen's University, Kingston, Ontario, Canada
}

\begin{abstract}
Subjects saw lists of three, four, five, or six digits; at the same time, they said them aloud, read them silently, or spoke the alphabet aloud (articulatory suppression). Recognition memory was examined with a probe presented $170 \mathrm{msec}$ after the last item. Strong recency effects were obtained, with the serial position functions for the longer list lengths being parallel and linear; it is suggested that the linear set-size function frequently found in this paradigm can be predicted from parallel serial position functions. It was also found that, although errors increased following articulatory suppression, mean reaction times did not. A theory is put forward that articulatory suppression affects registration in memory for some items but does not affect the recognition time required for other items in the list; on the other hand, items early in the list suffer a loss of discriminability, due to the presence of later items, which is reflected in slower recognition times.
\end{abstract}

This study on short-term recognition had two purposes. The first purpose was to determine the effect of varying levels of articulation upon the short-term recognition of visually presented material. It has been known for some time that in short-term recall, voicing the items aloud increases recall as compared with silent reading (Murray, 1965), and saying something other than the target material can reduce recall as compared with silent reading (Murray, 1967). Recent research has shown that preventing subvocal articulation probably interferes with subvocal intralist rehearsal (Broadbent \& Broadbent, 1981a) and thereby affects particularly ordered recall (Richardson, 1984). Purely sensory memory (visual or auditory) can coexist with items coded in speech memory (Baddeley, Lewis, \& Vallar, 1984). However, if the articulatory suppression task involves some cognitive activity in addition to mere exercise of the vocal apparatus, working memory will also be involved and registration of the target items in visual or speech memory may be affected, particularly if the material is visual and must be recoded.

One purpose of the present study, therefore, was to extend these findings to the case of recognition: if articulatory suppression influences registration as well as rehearsal, then errors might occur even if recognition is tested after short delays. Because the customary paradigm for examining short-term recognition is that of Sternberg (1966), we used that paradigm, which involved collecting yes-no recognition data for different list lengths, including measures of errors and of reaction times. Initially,

This research was supported by Natural Science and Engineering Research Council of Canada Grant No. A8505 to the author. I am grateful to Maureen Freedman and Fatima Kheraj for research assistance, to Sylvia Hains for advice on computation, and to W. E. Hockley for discussion. Requests for reprints should be sent to D. J. Murray, Department of Psychology, Queen's University, Kingston, Ontario, Canada K7L 3N6. it was expected that conditions associated with the high error rates would also be associated with long reaction times, because usually in the Sternberg paradigm, reaction times and error rates increase with list length. Note, however, that there is a problem in interpreting the results of short-term recognition experiments with high error rates, and for this reason, it will not be stressed how the present results are related to serial scanning or parallel matching process models. Such models have been prevalent in the literature, but Townsend and Ashby (1983) showed that they are very hard to evaluate on the basis of reaction time data.

A second purpose of the study was to evaluate the theory of why the typical set-size function, a linear relationship between mean reaction time and list length, might be found at brief retention intervals. It should be noted that Sternberg himself used a 2-sec delay between presentation of the list and probe. Forrin and Cunningham (1973) did a systematic study of the effects of probe delay upon recognition time, plotted as a serial position function, and found that delays over about $1 \mathrm{sec}$ were associated with flat serial position functions, whereas briefer delays were associated with pronounced recency effects, the shortest reaction times being for the last items.

Typically the curve at short probe delays shows a slight primacy effect (reduced recognition time for the first item) and then a monotonic decline of reaction time on serial position. However, both in other studies carried out in our laboratory and in Hockley (1984, Figure 11), who used a 1-sec delay, there has been an indication that the serial position function, apart from the primacy effect, can sometimes be linear. If each target stimulus somehow reduced the clarity of the preceding stimulus by a constant amount (a process related to what Broadbent \& Broadbent, 1981b, have called "overwriting"), and if the reaction time were related to the clarity of the item in memory, then a linear serial position function might be 
obtained. This is particularly important, for it can be shown that if functions relating recognition time to serial position have the same slope $k$ and intercept for different list lengths, then the resulting set-size function obtained by taking the means of each list length will also be linear with a slope of $k / 2$. In Hockley's data, there was no list length $\times$ serial position interaction, suggesting that his linear serial position effects had parallel slopes.

The second purpose of this study therefore was to examine the slopes of the recency effects expected to occur with a brief probe delay.

\section{METHOD}

In this experiment, the two independent variables were level of articulation and list length. For the former, subjects read lists of digits aloud, read each item silently, or read each item silently while speaking the alphabet aloud. Lists were three, four, five, or six digits long.

\section{Procedure}

Lists of three, four, five, or six digits were presented on the viewing screen of a Commodore 8K microcomputer; each digit appeared for $250 \mathrm{msec}$ with an interstimulus interval of $170 \mathrm{msec}$. At the start of a trial, the word READY appeared for $2,000 \mathrm{msec}$, followed by the digit sequence, followed by a probe digit that appeared $170 \mathrm{msec}$ after the last item. The probability that the digit had been present in the list was programmed to be .5 , and the program specified that each serial position in a list should be probed with equal probability. Immediately after the last digit of the list, a beep tone was sounded, signaling the end of the list. The subject responded by pressing a "Yes" key if he or she thought that the probe had been present in the list, and pressed a "No" key otherwise. (These keys were on the numerical pad of the keyboard.) Half of the subjects used the first finger of the right hand to signal "yes" and the second finger of the right hand to signal "no." These finger assignments were reversed for the remaining subjects. After the subjects responded, the next trial started automatically after $3,500 \mathrm{msec}$. Subjects participated in nine experimental sessions, three a week on consecutive days. Each session comprised 240 trials in which list lengths were randomly selected by the computer from trial to trial.

The three sessions of each week were devoted to one of the three conditions of articulation, so that over the nine sessions, each subject had 720 trials on each articulation condition. Under the voice condition, the subject spoke each item aloud as it appeared (excluding the probe). Under the read condition, the subject silently read each item as it appeared. Under the alphabet condition, the subject was given a letter of the alphabet when the READY sign came on and he or she spoke the alphabet aloud, starting at that letter, and beginning again at A if necessary, until the tone sounded at the end of the list. The subject was instructed to speak smoothly without interruption. The order in which these conditions were done, over the 3 weeks, was counterbalanced across subjects. On Day 1 of each of the conditions, 40 practice trials were given, and, on the succeeding 2 days, there were 16 initial practice trials.

\section{Subjects}

Subjects were 8 volunteer undergraduates taking psychology courses at Queen's University. They were paid for their services.

\section{RESULTS}

Figure 1a shows the serial position error functions for each list length, and Figure 1b shows the corresponding reaction time functions. The mean percentage error for the voice condition was 2.013 , for the read condition 3.297 , and for the alphabet condition 11.028. Analysis of variance on the overall array of error data (excluding the variable of serial position) showed that this effect was (a) PERCENTAGE ERRORS

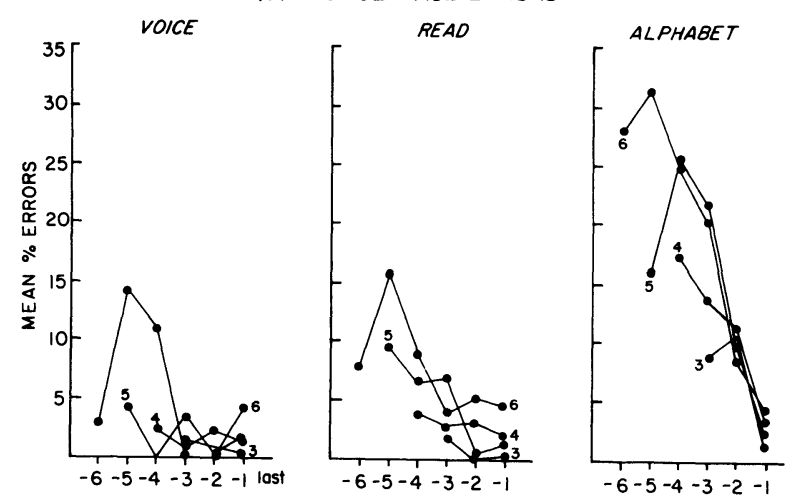

(b) REACTION TIMES
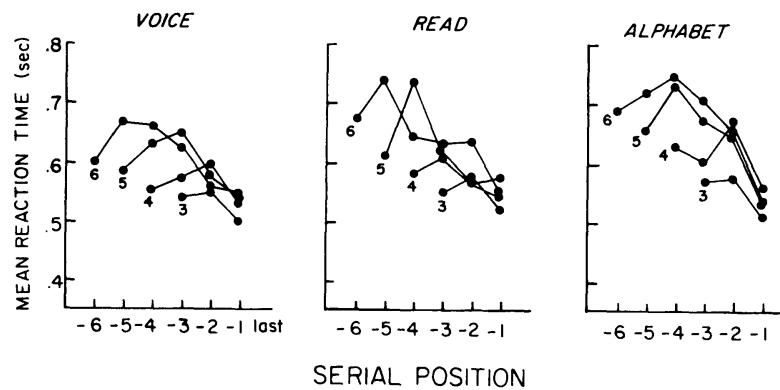

Figure 1. (a) Errors as a function of articulation condition, serial position, and list length; (b) mean reaction times as a function of articulation condition, serial position, and list length.

statistically significant $[F(2,14)=31.32, p<.001]$. A four-way ANOVA, also excluding serial position, in which the variables were articulatory condition, days, list length, and yes-no responding, was then applied to the reaction time data. This analysis showed that the effect of articulation level was no longer significant; performance improved across days $[F(2,14)=19.53$, $p<.0002]$; "yes" responses were faster than "no" responses $[F(1,7)=11.49, p<.01]$; and analysis on this last variable showed that the increase with list length had a significant linear component $[F(1,7)=45.51$, $p<.0005]$. Quadratic and cubic components were not significant. This typical set-size function is shown in Figure $2 b$. The only significant interaction in this analysis was days $\times$ list length $\times$ articulation $[F(12,84)=$ $2.53, p=.007]$. This interaction is shown in Figure 3, where we see that practice effects were the greatest in the alphabet condition, particularly for List Lengths 3 and 6 . Analysis of variance concerning serial position effects is complicated by the fact that lists were of different lengths; but if we examine reaction times for the last four serial positions only of List Lengths 4,5 , and 6 , we find a significant effect of serial position $[F(3,21)=17.39$, $p<.0001]$, as shown in Figure 2a.

Earlier, it was suggested that a linear set-size function could result if the serial position functions for each list length were linear and of equal slope and intercept. Figure 2a shows that one can join the points for the first 
(a)

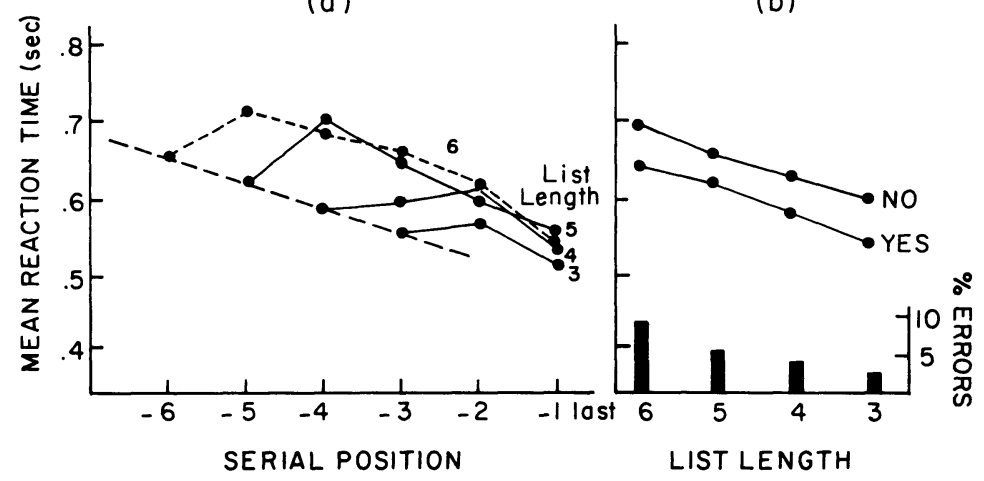

Figure 2. Mean reaction times as a function (a) of serial position and list length and (b) of list length, adding across serial positions. The dashed line in (a) is explained in the text.
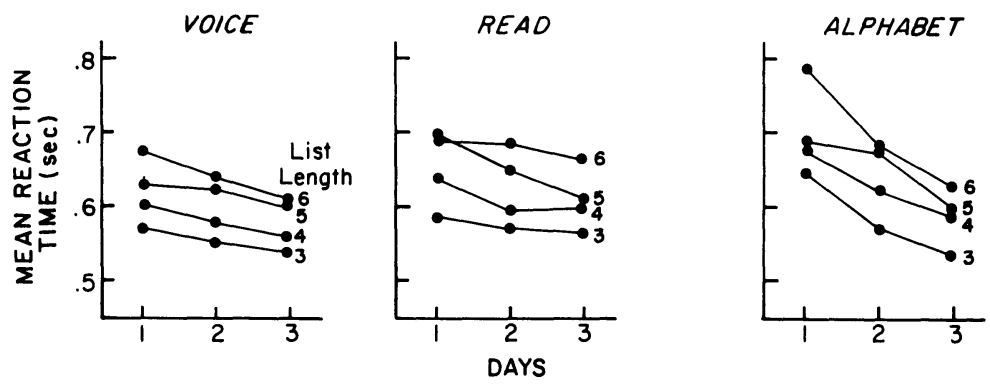

Figure 3. Mean reaction times as a function of articulation condition, list length, and days of practice.

position in each line and obtain a linear function, while it may be noted that, excluding the first position which showed a primacy effect, the serial position functions for List Lengths 5 and 6 could be fitted with straight lines associated with $r$ values of .996 and .941 , respectively ( $p<.001$ in both cases). The slope of the line best fitting the data points for List Length 5 was $49 \mathrm{msec}$, and that for List Length 6 was $41 \mathrm{msec}$, yielding a mean for the two of $44.8 \mathrm{msec}$; from this we predict the slope of the line joining the points for List Lengths 5 and 6 in the set-size function of Figure $2 \mathrm{~b}$ to be $22.4 \mathrm{msec}$ assuming a constant primacy effect. The slope was actually $22 \mathrm{msec}$.

\section{DISCUSSION}

Taken together with previous findings, these results seem to support the hypothesis that a linear set-size function following a brief delay can be seen as an outcome of the fact that serial position functions can be colinear. In fact, even if individual serial position curves are not linear, but lie parallel to each other, a linear set-size function could emerge. The important question is why the slope of the serial position function should change, and I suggest that at brief probe delays, overwriting causes a lack of clarity (i.e., a loss of discriminability, as might be expected on a distributed memory model such as that of Murdock, 1982). At longer probe delays there is time for rehearsal, as is also the case in the fixedset paradigm, so that items in short-term store are of approximately equal discriminability. However, even though serial position functions are flat, they do not necessarily have the same intercept, and the problem is to find an explanation for this which accounts for the linear set-size function. Examples of such flat serial position functions at longer probe delays can be found in Hockley (1984, Figure 3).

The results following the manipulation of articulation were unexpected. The pattern of errors was as predicted from previous work; the particularly high rate of errors following concurrent alphabet reciting suggests that both registration and intralist rehearsal were being interfered with. But why should reaction times not also change with level of articulation? If both errors and reaction times increased with the distance from the end of the list, why did they not both increase as articulation was reduced?

One possible explanation is that all comparisons between probe and target items were made on the basis of visual memory, and that articulation level was essentially a superfluous activity not affecting recognition time. But according to this argument, errors should also not have been affected by articulatory suppression, but they clearly were. This argument, therefore, is open to question.

A second possible explanation is that, as articulation was suppressed and working memory was occupied with reciting the alphabet, there were failures of registration of individual visually presented digits in visual, speech, and/or working memory. But the subject would realize that he or she had missed an item. Suppose, for example, that the digits in the list were $3,7,9$, and 5 , and because of suppression activity, the 9 did not register in memory. If 7 were the probe, it should activate Target 7 (possibly as in Glass's, 1984, parallel activation model), and the failure of the 9 to have registered would not affect the time required to say "yes"' to Probe 7. Even if a scanning procedure took place, the 9 would be processed as an unnamed event with no difference being found between the time to scan for the 7 if the 9 had or had not been registered in short-term memory. Thus, the reaction time to give a successful response need not vary if unregistered items are processed as if they were registered items with no names. On the other hand, if the 
probe had been 9, the subject would not know whether "nine" was the name of the unregistered item and would be forced to guess, yielding a high error rate for cases where probes correspond to unregistered items. Such cases would not be included as hits and, therefore, would not affect the mean reaction time to giving correct "yes" responses. However, there would be a few lucky guesses of "yes" on these occasions, but their number would probably be small.

This argument suggests that there are two kinds of unclear representation in this task. One kind is where the subject knew there had been an item but did not register its name. Such cases occur particularly under conditions of articulatory suppression and yield high error rates but do not affect mean reaction times to retrieve other items in the list. The second kind is due to overwriting, where an item is less discriminable because new items have entered memory. In this case, direct access by the probe is not always possible and the subject may have to search, scan, or otherwise bring the item into clear consciousness before the match can be made with the probe. It is also possible that an automatic process of correlation between target item and probe is rendered slower by a lack of discriminability between the target item and other target items, as Murdock (1982) has suggested. Whatever the explanation, items which have been overwritten are associated with both high error rates and long reaction times.

It is suggested then that articulatory suppression has an effect on the registration process, whereas overwriting has an effect on the discriminability of a target item in memory. One effect of the presence of overwriting is that there will be a serial position function for reaction times which increases with distance from the end of the list. If the slope of this function is the same for several list lengths, it is possible to predict the set-size function.

\section{REFERENCES}

Baddeley, A. D., Lewis, V. \& Vallar, G. (1984). Exploring the articulatory loop. Quarterly Journal of Experimental Psychology, 36A, 233-252.
Broadbent, D. E., \& Broadbent, M. H. P. (1981a). Articulatory suppression and the grouping of successive stimuli. Psychological Research, 43, 53-67.

Broadbent, D. E., \& Broadbent, M. H. P. (1981b). Recency effects in visual memory. Quarterly Journal of Experimental Psychology, 33A, 1-15.

ForRIN, B., \& CunNingham, K. (1973). Recognition time and serial position of probed item in short-term memory. Journal of Experimental Psychology, 99, 272-279.

Glass, A. L. (1984). Effect of memory set on reaction time. In J. R. Anderson \& S. M. Kosslyn (Eds.), Tutorials in learning and memory. San Francisco: Freeman.

HoCKLEY, W. E. (1984). Analysis of response time distributions in the study of cognitive processes. Journal of Experimental Psychology: Learning, Memory, \& Cognition, 10, 598-615.

MuRDOCK, B. B., JR. (1982). A theory for the storage and retrieval of item and associative information. Psychological Review, 89, 609-626.

MURRAY, D. J. (1965). Vocalization-at-presentation and immediate recall, with varying presentation rates. Quarterly Journal of Experimental Psychology, 17, 47-56.

MuRRaY, D. J. (1967). The role of speech responses in short-term memory. Canadian Journal of Psychology, 21, 263-276.

Richardson, J. T. E. (1984). Developing the theory of working memory. Memory \& Cognition, 12, 71-83.

STERNBERG, S. (1966). High-speed scanning in human memory. Science, 153, 652-654.

TownSEND, J. T., \& AshBY, F. G. (1983). The stochastic modeling of elementary psychology processes. New York: Cambridge University Press.

(Manuscript received for publication November 2, 1985.) 